\title{
A SHARP INEQUALITY FOR HOLOMORPHIC FUNCTIONS ON THE POLYDISC
}

\author{
MARIJAN MARKOVIĆ \\ (Communicated by Franc Forstneric)
}

\begin{abstract}
In this paper we prove an isoperimetric inequality for holomorphic functions in the unit polydisc $\mathbf{U}^{n}$. As a corollary we derive an inclusion relation between weighted Bergman and Hardy spaces of holomorphic functions in the polydisc which generalizes the classical Hardy-Littlewood relation $H^{p} \subseteq A^{2 p}$. Also, we extend some results due to Burbea.
\end{abstract}

\section{IntRoduction AND STATEMENT OF THE RESUlt}

1.1. Notation. For an integer $n \geq 1$ we consider the $n$-dimensional complex vector space $\mathbf{C}^{n}$ with the usual inner product

$$
\langle z, \zeta\rangle=\sum_{j=1}^{n} z_{j} \bar{\zeta}_{j}, \quad z, \zeta \in \mathbf{C}^{n}
$$

and norm

$$
\|z\|=\langle z, z\rangle^{\frac{1}{2}}
$$

where $z=\left(z_{1}, \ldots, z_{n}\right), \zeta=\left(\zeta_{1}, \ldots, \zeta_{n}\right), \mathbf{U}$ is the open unit disc in the complex plane $\mathbf{C}, \mathbf{T}$ is its boundary, and $\mathbf{U}^{n}$ and $\mathbf{T}^{n}$ stand for the unit polydisc and its distinguished boundary, respectively.

Following the classical book of Rudin 31, let us recall some basic facts from the theory of Hardy spaces $H^{p}\left(\mathbf{U}^{n}\right)$ on the unit polydisc. Let $p>0$ be an arbitrary real (in the sequel the letter $p$, with or without index, will stand for a positive real number if we do not make restrictions). By $d m_{n}$ we denote the Haar measure on the distinguished boundary $\mathbf{T}^{n}$, i.e.,

$$
d m_{n}(\omega)=\frac{1}{(2 \pi)^{n}} d \theta_{1} \ldots d \theta_{n}, \quad \omega=\left(e^{i \theta_{1}}, \ldots, e^{i \theta_{n}}\right) \in \mathbf{T}^{n} .
$$

A holomorphic function $f$ in the polydisc $\mathbf{U}^{n}$ belongs to the Hardy space $H^{p}\left(\mathbf{U}^{n}\right)$ if it satisfies the growth condition

$$
\|f\|_{H^{p}\left(\mathbf{U}^{n}\right)}:=\left(\sup _{0 \leq r<1} \int_{\mathbf{T}^{n}}|f(r \omega)|^{p} d m_{n}(\omega)\right)^{\frac{1}{p}}<\infty .
$$

Received by the editors May 27, 2011 and, in revised form, August 9, 2011 and September 9, 2011.

2010 Mathematics Subject Classification. Primary 30H10, 32A36, 30A10.

Key words and phrases. Weighted Bergman spaces, Hardy spaces, isoperimetric inequality, polydisc. 
It turns out that if $f \in H^{p}\left(\mathbf{U}^{n}\right)$, then there exists

$$
\lim _{r \rightarrow 1} f(r \omega)=f(\omega) \text { a.e. on } \mathbf{T}^{n}
$$

and the boundary function belongs to $L^{p}\left(\mathbf{T}^{n}, m_{n}\right)$, the Lebesgue space of all $p$ integrable functions on $\mathbf{T}^{n}$ (with respect to the measure $m_{n}$ ). Moreover,

$$
\int_{\mathbf{T}^{n}}|f(\omega)|^{p} d m_{n}(\omega)=\sup _{0 \leq r<1} \int_{\mathbf{T}^{n}}|f(r \omega)|^{p} d m_{n}(\omega) .
$$

For $q>-1$ let

$$
d \mu_{q}(z)=\frac{(q+1)}{\pi}\left(1-|z|^{2}\right)^{q} d x d y \quad(z=x+i y \in \mathbf{U})
$$

stand for a weighted normalized measure on the disc $\mathbf{U}$. We will consider also the corresponding measure on the polydisc $\mathbf{U}^{n}$,

$$
d \mu_{\mathbf{q}}(z)=\prod_{k=1}^{n} d \mu_{q_{k}}\left(z_{k}\right), \quad z \in \mathbf{U}^{n}
$$

where $\mathbf{q}>-\mathbf{1}$ is an $n$-multi-index; the inequality $\mathbf{q}_{1}>\mathbf{q}_{2}$ between two $n$-multiindices means that $q_{1, k}>q_{2, k}, k=1, \ldots, n$; we denote the $n$-multi-index $(m, \ldots, m)$ by $\mathbf{m}$. For a real number $m, m>1$, we have

$$
d \mu_{\mathbf{m}-\mathbf{2}}(z)=\frac{(m-1)^{n}}{\pi^{n}} \prod_{k=1}^{n}\left(1-\left|z_{k}\right|^{2}\right)^{m-2} d x_{k} d y_{k} \quad\left(z_{k}=x_{k}+i y_{k}\right) .
$$

The weighted Bergman spaces $A_{\mathbf{q}}^{p}\left(\mathbf{U}^{n}\right), p>0, \mathbf{q}>-\mathbf{1}$ contain the holomorphic functions $f$ in the polydisc $\mathbf{U}^{n}$ such that

$$
\|f\|_{A_{\mathbf{q}}^{p}\left(\mathbf{U}^{n}\right)}:=\left(\int_{\mathbf{U}^{n}}|f(z)|^{p} d \mu_{\mathbf{q}}(z)\right)^{\frac{1}{p}}<\infty .
$$

Since $d \mu_{0}$ is the area measure on the complex plane normalized on the unit disc, $A^{p}\left(\mathbf{U}^{n}\right):=A_{\mathbf{0}}^{p}\left(\mathbf{U}^{n}\right)$ are the ordinary (unweighted) Bergman spaces on $\mathbf{U}^{n}$.

It is well known that $\|\cdot\|_{H^{p}\left(\mathbf{U}^{n}\right)}$ and $\|\cdot\|_{A_{\mathbf{q}}^{p}\left(\mathbf{U}^{n}\right)}$ are norms on $H^{p}\left(\mathbf{U}^{n}\right)$ and $A_{\mathbf{q}}^{p}\left(\mathbf{U}^{n}\right)$, respectively, if $p \geq 1$, and quasi-norms for $0<p<1$; for simplicity, we sometimes write $\|\cdot\|_{p}$ and $\|\cdot\|_{p, \mathbf{q}}$. As usual, $H^{p}(\mathbf{U})$ and $A_{q}^{p}(\mathbf{U})$ are denoted by $H^{p}$ and $A_{q}^{p}$. Obviously, $H^{p}\left(\mathbf{U}^{n}\right) \subseteq A_{\mathbf{q}}^{p}\left(\mathbf{U}^{n}\right)$.

Let us point out that the Hardy space $H^{2}\left(\mathbf{U}^{n}\right)$ is a Hilbert space with the reproducing kernel

$$
K_{n}(z, \zeta)=\prod_{j=1}^{n} \frac{1}{1-z_{j} \bar{\zeta}_{j}}, \quad z, \zeta \in \mathbf{U}^{n}
$$

For the theory of reproducing kernels we refer to 1 .

1.2. Short background. The solution to the isoperimetric problem is usually expressed in the form of an inequality that relates the length $L$ of a closed curve and the area $A$ of the planar region that it encloses. The isoperimetric inequality states that

$$
4 \pi A \leq L^{2},
$$

and that equality holds if and only if the curve is a circle. Dozens of proofs of the isoperimetric inequality have been proposed. More than one approach can be found in the expository papers by Osserman [26, Gamelin and Khavinson [10] and 
Bläsjö [6] along with a brief history of the problem. For a survey of some known generalizations to higher dimensions and the list of some open problems, we refer to the paper by Bénéteau and Khavinson [3].

In [7, Carleman gave a beautiful proof of the isoperimetric inequality in the plane, reducing it to an inequality for holomorphic functions in the unit disc. Following Carleman's result, Aronszajn in [1 showed that if $f_{1}$ and $f_{2}$ are holomorphic functions in a simply connected domain $\Omega$ with analytic boundary $\partial \Omega$, such that $f_{1}, f_{2} \in H^{2}(\Omega)$, then

$$
\int_{\Omega}\left|f_{1}\right|^{2}\left|f_{2}\right|^{2} d x d y \leq \frac{1}{4 \pi} \int_{\partial \Omega}\left|f_{1}\right|^{2}|d z| \int_{\partial \Omega}\left|f_{2}\right|^{2}|d z| \quad(z=x+i y) .
$$

In 14, Jacobs considered not only simply connected domains, but also multiply connected domains (see also the Saitoh work 32]).

Mateljević and Pavlović in 23 generalized (1.3) in the following sense: if $f_{j} \in$ $H^{p_{j}}(\Omega), j=1,2$, where $\Omega$ is a simply connected domain with analytic boundary $\partial \Omega$, then

$$
\frac{1}{\pi} \int_{\Omega}\left|f_{1}\right|^{p_{1}}\left|f_{2}\right|^{p_{2}} d x d y \leq \frac{1}{4 \pi^{2}} \int_{\partial \Omega}\left|f_{1}\right|^{p_{1}}|d z| \int_{\partial \Omega}\left|f_{2}\right|^{p_{2}}|d z|,
$$

with equality if and only if either $f_{1} f_{2} \equiv 0$ or if for some $C_{j} \neq 0$,

$$
f_{j}=C_{j}\left(\psi^{\prime}\right)^{\frac{1}{p_{j}}}, \quad j=1,2,
$$

where $\psi$ is a conformal mapping of the domain $\Omega$ onto the disc $\mathbf{U}$.

By using a similar approach as Carleman, Strebel in his book 33, Theorem 19.9, pp. 96-98] (see also the papers [21] and 34]) proved that if $f \in H^{p}$, then

$$
\int_{\mathbf{U}}|f(z)|^{2 p} d x d y \leq \frac{1}{4 \pi}\left(\int_{0}^{2 \pi}\left|f\left(e^{i \theta}\right)\right|^{p} d \theta\right)^{2} \quad\left(\|f\|_{A^{2 p}} \leq\|f\|_{H^{p}}\right),
$$

with equality if and only if for some constants $\zeta,|\zeta|<1$ and $\lambda$,

$$
f(z)=\frac{\lambda}{(1-z \bar{\zeta})^{\frac{2}{p}}} .
$$

Further, Burbea in [5] generalized (1.5]) to

$$
\frac{m-1}{\pi} \int_{\mathbf{U}}|f(z)|^{m p}\left(1-|z|^{2}\right)^{m-2} d x d y \leq\left(\frac{1}{2 \pi} \int_{0}^{2 \pi}\left|f\left(e^{i \theta}\right)\right|^{p} d \theta\right)^{m},
$$

where $m \geq 2$ is an integer. The equality is attained in the same case as in the relation (1.5). The inequality (1.6) can be rewritten as

$$
\|f\|_{A_{m-1}^{m p}} \leq\|f\|_{H^{p}}, \quad f \in H^{p},
$$

which is a generalization of the inclusion $H^{p} \subseteq A^{2 p}$, proved by Hardy and Littlewood in 12 .

On the other hand, Pavlović and Dostanić showed in 28] that if $\mathbf{B}_{n}$ is the unit ball in $\mathbf{C}^{n}, \partial \mathbf{B}_{n}$ is its boundary, and $\sigma_{n}$ is the normalized surface area measure on the sphere $\partial \mathbf{B}_{n}$, then

$$
\int_{\partial \mathbf{B}_{n}}|f|^{2 n} d \sigma_{n} \leq\left(\int_{\mathbf{T}^{n}}|f|^{2} d m_{n}\right)^{n}
$$


holds for $f \in H^{2}\left(\mathbf{U}^{n}\right)$. They pointed out that this inequality coincides with (1.6) for $m=n, p=2$ and $f(z)=f\left(z_{1}, \ldots, z_{n}\right)=f\left(z_{1}\right)$, that is, if $f$ actually depends only on one complex variable.

For an isoperimetric inequality for harmonic functions we refer to [16].

1.3. Statement of the result. In the sequel, $m$ stands for an integer $\geq 2$. The starting point of this paper is the work of Burbea [5], who obtained the following isoperimetric inequalities concerning the unit disc and the unit polydisc.

Proposition 1.1. Let $f_{j} \in H^{p_{j}}, j=1, \ldots, m$. Then

$$
\int_{\mathbf{U}} \prod_{j=1}^{m}\left|f_{j}\right|^{p_{j}} d \mu_{m-2} \leq \prod_{j=1}^{m} \int_{\mathbf{T}}\left|f_{j}\right|^{p_{j}} d m_{1} .
$$

Equality holds if and only if either some of the functions are identically equal to zero or if for some point $\zeta \in \mathbf{U}$ and constants $C_{j} \neq 0$,

$$
f_{j}=C_{j} K_{1}^{\frac{2}{p_{j}}}(\cdot, \zeta), \quad j=1, \ldots, m,
$$

where $K_{1}$ is the reproducing kernel (1.2) for the Hardy space $H^{2}$.

Proposition 1.2. Let $f_{j} \in H^{2}\left(\mathbf{U}^{n}\right), j=1, \ldots, m$. Then

$$
\int_{\mathbf{U}^{n}} \prod_{j=1}^{m}\left|f_{j}\right|^{2} d \mu_{\mathbf{m}-\mathbf{2}} \leq \prod_{j=1}^{m} \int_{\mathbf{T}^{n}}\left|f_{j}\right|^{2} d m_{n} .
$$

Equality holds if and only if either some of the functions are identically equal to zero or if for some point $\zeta \in \mathbf{U}^{n}$ and constants $C_{j} \neq 0$,

$$
f_{j}=C_{j} K_{n}(\cdot, \zeta), \quad j=1, \ldots, m,
$$

where $K_{n}$ is the reproducing kernel (1.2).

Proposition 1.2 is a particular case of Theorem 4.1 in the Burbea paper [5, p. 257]. That theorem was derived from more general considerations involving the theory of reproducing kernels (see also 4]). The inequality in that theorem is between Bergman type norms, while Proposition 1.2 is the case with the Hardy norm on the right side (in that case, we have an isoperimetric inequality). In the next main theorem we extend (1.7) for holomorphic functions which belong to general Hardy spaces on the polydisc $\mathbf{U}^{n}$.

Theorem 1.3. Let $f_{j} \in H^{p_{j}}\left(\mathbf{U}^{n}\right), j=1, \ldots, m$. Then

$$
\int_{\mathbf{U}^{n}} \prod_{j=1}^{m}\left|f_{j}\right|^{p_{j}} d \mu_{\mathbf{m}-\mathbf{2}} \leq \prod_{j=1}^{m} \int_{\mathbf{T}^{n}}\left|f_{j}\right|^{p_{j}} d m_{n} .
$$

Equality occurs if and only if either some of the functions are identically equal to zero or if for some point $\zeta \in \mathbf{U}^{n}$ and constants $C_{j} \neq 0$,

$$
f_{j}=C_{j} K_{n}^{\frac{2}{p_{j}}}(\cdot, \zeta), \quad j=1, \ldots, m .
$$

Notice that in higher complex dimensions there is no analog of the Blaschke product, so we cannot deduce Theorem 1.3 directly from Proposition 1.2 as we can for $n=1$ (this is a usual approach in the theory of $H^{p}$ spaces; see also [5]). We will prove the main theorem in the case $n=2$ since for $n>2$ our method needs only a technical adaptation. As immediate consequences of Theorem 1.3. we have the next two corollaries. 
Corollary 1.4. Let $p \geq 1$. The (polylinear) operator $\beta: \bigotimes_{j=1}^{m} H^{p}\left(\mathbf{U}^{n}\right) \rightarrow$ $A_{\mathbf{m}-\mathbf{2}}^{p}\left(\mathbf{U}^{n}\right)$, defined by $\beta\left(f_{1}, \ldots, f_{m}\right)=\prod_{j=1}^{m} f_{j}$, has norm one.

Corollary 1.5. Let $f \in H^{p}\left(\mathbf{U}^{n}\right)$. Then

$$
\int_{\mathbf{U}^{n}}|f|^{m p} d \mu_{\mathbf{m}-\mathbf{2}} \leq\left(\int_{\mathbf{T}^{n}}|f|^{p} d m_{n}\right)^{m} .
$$

Equality occurs if and only if for some point $\zeta \in \mathbf{U}^{n}$ and constant $\lambda$,

$$
f=\lambda K_{n}^{\frac{2}{p}}(\cdot, \zeta)
$$

In other words, we have the sharp inequality

$$
\|f\|_{A_{\mathbf{m}-\mathbf{2}}^{m p}\left(\mathbf{U}^{n}\right)} \leq\|f\|_{H^{p}\left(\mathbf{U}^{n}\right)}, \quad f \in H^{p}\left(\mathbf{U}^{n}\right),
$$

and the inclusion

$$
H^{p}\left(\mathbf{U}^{n}\right) \subseteq A_{\mathbf{m}-\mathbf{2}}^{m p}\left(\mathbf{U}^{n}\right) .
$$

Thus, when $p \geq 1$, the inclusion map $I_{p, m}: H^{p}\left(\mathbf{U}^{n}\right) \rightarrow A_{\mathbf{m}-\mathbf{2}}^{m p}\left(\mathbf{U}^{n}\right), I_{p, m}(f):=f$, has norm one.

\section{Proof OF THE MAIN THEOREM}

Following Beckenbach and Radó [2, we say that a nonnegative function $u$ is logarithmically subharmonic in a plane domain $\Omega$ if $u \equiv 0$ or if $\log u$ is subharmonic in $\Omega$.

Our first step is to extend the Burbea inequality (1.7) to functions which belong to the spaces $h_{P L}^{p}$ defined in the following sense: $u \in h_{P L}^{p}$ if it is logarithmically subharmonic and satisfies the growth property

$$
\sup _{0 \leq r<1} \int_{0}^{2 \pi}\left|u\left(r e^{i \theta}\right)\right|^{p} \frac{d \theta}{2 \pi}<\infty .
$$

It is known that a function from these spaces has a radial limit in $e^{i \theta} \in \mathbf{T}$ for almost all $\theta \in[0,2 \pi]$. Let us denote this limit (when it exists) as $u\left(e^{i \theta}\right)$. Then $\lim _{r \rightarrow 1} \int_{0}^{2 \pi}\left|u\left(r e^{i \theta}\right)\right|^{p} \frac{d \theta}{2 \pi}=\int_{0}^{2 \pi}\left|u\left(e^{i \theta}\right)\right|^{p} \frac{d \theta}{2 \pi}$ and $\lim _{r \rightarrow 1} \int_{0}^{2 \pi}\left|u\left(r e^{i \theta}\right)-u\left(e^{i \theta}\right)\right|^{p} \frac{d \theta}{2 \pi}=0$. For an exposition of the topic of spaces of logarithmically subharmonic functions which satisfy (2.1), we refer to the book of Privalov [29].

Lemma 2.1. Let $u_{j} \in h_{P L}^{p_{j}}, j=1, \ldots, m$, be logarithmically subharmonic functions in the unit disc. Then

$$
\int_{\mathbf{U}} \prod_{j=1}^{m} u_{j}^{p_{j}} d \mu_{m-2} \leq \prod_{j=1}^{m} \int_{\mathbf{T}} u_{j}^{p_{j}} d m_{1} .
$$

For continuous functions, equality holds if and only if either some of the functions are identically equal to zero or if for some point $\zeta \in \mathbf{U}$ and constants $\lambda_{j}>0$,

$$
u_{j}=\lambda_{j}\left|K_{1}(\cdot, \zeta)\right|^{\frac{2}{p_{j}}}, \quad j=1, \ldots, m \text {. }
$$

Proof. Suppose that no one of the functions $u_{j}, j=1, \ldots, m$ is identically equal to zero. Then $\log u_{j}\left(e^{i \theta}\right)$ is integrable on the segment $[0,2 \pi]$ and there exist $f_{j} \in H^{p_{j}}$ such that $u_{j}(z) \leq\left|f_{j}(z)\right|, z \in \mathbf{U}$ and $u_{j}\left(e^{i \theta}\right)=\left|f_{j}\left(e^{i \theta}\right)\right|, \theta \in[0,2 \pi]$. Namely, for $f_{j}$ we can choose

$$
f_{j}(z)=\exp \left(\int_{0}^{2 \pi} \frac{e^{i \theta}+z}{e^{i \theta}-z} \log u_{j}\left(e^{i \theta}\right) \frac{d \theta}{2 \pi}\right), \quad j=1, \ldots, m .
$$


Since $\log u_{j}$ is subharmonic we have

$$
\log u_{j}(z) \leq \int_{0}^{2 \pi} P\left(z, e^{i \theta}\right) \log u_{j}\left(e^{i \theta}\right) \frac{d \theta}{2 \pi},
$$

where $P\left(z, e^{i \theta}\right)=\frac{1-|z|^{2}}{\left|z-e^{i \theta}\right|^{2}}$ is the Poisson kernel for the disc $\mathbf{U}$. From this it follows that $u_{j}(z) \leq\left|f_{j}(z)\right|, z \in \mathbf{U}$. Moreover, using the Jensen inequality (for the concave function $\log$ ) we obtain

$$
\begin{aligned}
\log \left|f_{j}(z)\right|^{p_{j}} & =\int_{0}^{2 \pi} P\left(z, e^{i \theta}\right) \log \left|u_{j}\left(e^{i \theta}\right)\right|^{p_{j}} \frac{d \theta}{2 \pi} \\
& \leq \log \int_{0}^{2 \pi} P\left(z, e^{i \theta}\right) u_{j}^{p_{j}}\left(e^{i \theta}\right) \frac{d \theta}{2 \pi}
\end{aligned}
$$

implying $f_{j} \in H^{p_{j}}$.

Now, in (1.7) we can take $f_{j}, j=1, \ldots, m$, and use the previous relations, $u_{j}(z) \leq\left|f_{j}(z)\right|, z \in \mathbf{U}$ and $u_{j}\left(e^{i \theta}\right)=\left|f_{j}\left(e^{i \theta}\right)\right|, \theta \in[0,2 \pi]$, to derive the inequality (2.2).

If all of the functions $u_{j}, j=1, \ldots, m$ are continuous (not equal to zero identically) and if the equality in (2.2) occurs, then $u_{j}(z)=\left|f_{j}(z)\right|, z \in \mathbf{U}, j=1, \ldots, m$. According to the equality in Proposition 1.1 we must have $f_{j}=C_{j} K_{1}^{\frac{2}{p_{j}}}(\cdot, \zeta), j=$ $1, \ldots, m$, for some point $\zeta \in \mathbf{U}$ and constants $C_{j} \neq 0$. Thus, for continuous functions (not equal to zero identically), equality holds if and only if $u_{j}=\lambda_{j}\left|K_{1}(\cdot, \zeta)\right|^{\frac{2}{p_{j}}}$, $j=1, \ldots, m\left(\lambda_{j}>0\right)$.

We need the next two propositions concerning (logarithmically) subharmonic functions. For the proofs of these propositions, see the first paragraph of the book of Ronkin [30].

Proposition 2.2. Let $f$ be an upper semi-continuous function on a product $\Omega \times \Delta$ of domains $\Omega \subseteq \mathbf{R}^{n}$ and $\Delta \subseteq \mathbf{R}^{k}$. Let $\mu$ be a positive measure on $\Delta$ and $E \subseteq \Delta$ be such that $\mu(E)<\infty$. Then

$$
\varphi(x):=\int_{E} f(x, y) d \mu(y), \quad x \in \Omega,
$$

is (logarithmically) subharmonic if $f(\cdot, y)$ is (logarithmically) subharmonic for all (almost all with respect to the measure $\mu$ ) $y \in \Omega$.

Proposition 2.3. Let $A$ be an index set and $\left\{u_{\alpha}, \alpha \in A\right\}$ a family of (logarithmically) subharmonic functions in a domain $\Omega \subseteq \mathbf{R}^{n}$. Then

$$
u(x):=\sup _{\alpha \in A} u_{\alpha}(x), \quad x \in \Omega,
$$

is (logarithmically) subharmonic if it is upper semi-continuous in the domain $\Omega$.

Also, we need the next theorem, due to Vitali (see [11]).

Theorem 2.4 (Vitali). Let $X$ be a measurable space with finite measure $\mu$, and let $h_{n}: X \rightarrow \mathbf{C}$ be a sequence of functions that are uniformly integrable, i.e., such that for every $\epsilon>0$ there exists $\delta>0$, independent of $n$, satisfying

$$
\mu(E)<\delta \Rightarrow \int_{E}\left|h_{n}\right| d \mu<\epsilon
$$


Then if $h_{n}(x) \rightarrow h(x)$ a.e.,

$$
\lim _{n \rightarrow \infty} \int_{X}\left|h_{n}\right| d \mu=\int_{X}|h| d \mu .
$$

In particular, if $\sup _{n} \int_{X}\left|h_{n}\right| d \mu<\infty$, then the previous condition holds.

Lemma 2.5. Let $f \in H^{p}\left(\mathbf{U}^{2}\right)$. Then

$$
\phi(z):=\int_{0}^{2 \pi}\left|f\left(z, e^{i \eta}\right)\right|^{p} \frac{d \eta}{2 \pi}
$$

is continuous. Moreover, $\phi$ is logarithmically subharmonic and belongs to the space $h_{P L}^{1}$.

Proof. For $0 \leq r<1$, let us denote

$$
\phi_{r}(z):=\int_{0}^{2 \pi}\left|f\left(z, r e^{i \eta}\right)\right|^{p} \frac{d \eta}{2 \pi}, \quad z \in \mathbf{U} .
$$

According to Proposition 2.2, $\phi_{r}$ is logarithmically subharmonic in the unit disc, since $z \rightarrow\left|f\left(z, r e^{i \eta}\right)\right|^{p}$ are logarithmically subharmonic for $\eta \in[0,2 \pi]$. Since for all $z \in \mathbf{U}$ we have $\phi_{r}(z) \rightarrow \phi(z)$, monotone as $r \rightarrow 1$, it follows that $\phi(z)=$ $\sup _{0 \leq r<1} \phi_{r}(z)$. Thus, we have only to prove (by Proposition 2.3) that $\phi$ is continuous.

First of all we have

$$
\phi(z)=\|f(z, \cdot)\|_{p} \leq C_{p}(1-|z|)^{-\frac{1}{p}}\|f\|_{p}, \quad z \in \mathbf{U},
$$

for some positive constant $C_{p}$. Namely, according to the theorem of Hardy and Littlewood (see [12, Theorem 27, or [8], Theorem 5.9) applied to the one-variable function $f(\cdot, w)$ with $w$ fixed, we obtain

$$
|f(z, w)| \leq C_{p}(1-|z|)^{-\frac{1}{p}}\|f(\cdot, w)\|_{p}, \quad(z, w) \in \mathbf{U}^{2},
$$

for some $C_{p}>0$. Using the above inequality and the monotone convergence theorem, we derive

$$
\begin{aligned}
\|f(z, \cdot)\|_{p}^{p} & =\lim _{s \rightarrow 1} \int_{0}^{2 \pi}\left|f\left(z, s e^{i \eta}\right)\right|^{p} \frac{d \eta}{2 \pi} \\
& \leq C_{p}^{p}(1-|z|)^{-1} \lim _{s \rightarrow 1} \int_{0}^{2 \pi}\left\|f\left(\cdot, s e^{i \eta}\right)\right\|_{p}^{p} \frac{d \eta}{2 \pi} \\
& =C_{p}^{p}(1-|z|)^{-1} \lim _{s \rightarrow 1} \int_{0}^{2 \pi} \frac{d \theta}{2 \pi} \lim _{r \rightarrow 1} \int_{0}^{2 \pi}\left|f\left(r e^{i \theta}, s e^{i \eta}\right)\right|_{p}^{p} \frac{d \eta}{2 \pi} \\
& =C_{p}^{p}(1-|z|)^{-1} \lim _{(r, s) \rightarrow(1,1)} \int_{0}^{2 \pi} \int_{0}^{2 \pi}\left|f\left(r e^{i \theta}, s e^{i \eta}\right)\right|_{p}^{p} \frac{d \theta}{2 \pi} \frac{d \eta}{2 \pi} \\
& =C_{p}^{p}(1-|z|)^{-1}\|f\|_{p}^{p},
\end{aligned}
$$

and (2.3) follows.

The inequality (2.3) implies that the family of integrals

$$
\left\{\phi(z)=\int_{0}^{2 \pi}\left|f\left(z, e^{i \eta}\right)\right|^{p} \frac{d \eta}{2 \pi}: z \in \mathbf{U}\right\}
$$

is uniformly bounded on compact subsets of the unit disc. Since $z \rightarrow\left|f\left(z, e^{i \eta}\right)\right|^{p}$ is continuous for almost all $\eta \in[0,2 \pi]$, as a module of a holomorphic function (according to [36, Theorem XVII, 5.16) it follows that $\phi(z), z \in \mathbf{U}$ is continuous. 
Indeed, let $z_{0} \in \mathbf{U}$ and let $\left(z_{k}\right)_{k \geq 1}$ be a sequence in the unit disc such that $z_{k} \rightarrow$ $z_{0}, k \rightarrow \infty$. According to the Vitali theorem we have

$$
\lim _{k \rightarrow \infty} \phi\left(z_{k}\right)=\lim _{k \rightarrow \infty} \int_{0}^{2 \pi}\left|f\left(z_{k}, e^{i \eta}\right)\right|^{p} \frac{d \eta}{2 \pi}=\int_{0}^{2 \pi}\left|f\left(z_{0}, e^{i \eta}\right)\right|^{p} \frac{d \eta}{2 \pi}=\phi\left(z_{0}\right) .
$$

We now prove the main Theorem 1.3 ,

Proof. Let $f_{j} \in H^{p_{j}}\left(\mathbf{U}^{2}\right), j=1, \ldots, m$ be holomorphic functions in the polydisc $\mathbf{U}^{2}$. Using the Fubini theorem, Proposition 1.1, and Lemma 2.1, we obtain

$$
\begin{aligned}
\int_{\mathbf{U}^{2}} \prod_{j=1}^{m}\left|f_{j}\right|^{p_{j}} d \mu_{(m-2, m-2)} & =\int_{\mathbf{U}} d \mu_{m-2}(z) \int_{\mathbf{U}} \prod_{j=1}^{m}\left|f_{j}(z, w)\right|^{p_{j}} d \mu_{m-2}(w) \\
& \leq \int_{\mathbf{U}} d \mu_{m-2}(z) \prod_{j=1}^{m} \int_{0}^{2 \pi}\left|f_{j}\left(z, e^{i \eta}\right)\right|^{p_{j}} \frac{d \eta}{2 \pi} \\
& \leq \prod_{j=1}^{m} \int_{0}^{2 \pi} \frac{d \theta}{2 \pi} \int_{0}^{2 \pi}\left|f_{j}\left(e^{i \theta}, e^{i \eta}\right)\right|^{p_{j}} \frac{d \eta}{2 \pi} \\
& =\prod_{j=1}^{m} \int_{\mathbf{T}^{2}}\left|f_{j}\right|^{p_{j}} d m_{2},
\end{aligned}
$$

since the functions $\phi_{j}(z):=\int_{0}^{2 \pi}\left|f_{j}\left(z, e^{i \eta}\right)\right|^{p_{j}} \frac{d \eta}{2 \pi}$ are logarithmically subharmonic in the disc $\mathbf{U}$ and since $\phi_{j} \in h_{P L}^{1}, j=1, \ldots, m$, by Lemma 2.5 .

We now determine when the equalities hold in the above inequalities. Obviously, if some of the functions $f_{j}, j=1, \ldots, m$ are identically equal to zero, we have equalities everywhere. Suppose this is not the case. We will first prove that the $f_{j}, j=1, \ldots, m$ do not vanish in the polydisc $\mathbf{U}^{2}$.

Since for $j=1, \ldots, m$ we have $\phi_{j} \not \equiv 0$, the equality is obtained in the second inequality if and only if for some point $\zeta^{\prime \prime} \in \mathbf{U}$ and $\lambda_{j}>0$ we have $\phi_{j}=$ $\lambda_{j}\left|K_{1}\left(\cdot, \zeta^{\prime \prime}\right)\right|^{2}, j=1, \ldots, m$. Thus, $\phi_{j}$ is free of zeroes in the unit disc. Let

$$
\psi(z):=\int_{\mathbf{U}} \prod_{j=1}^{m}\left|f_{j}(z, w)\right|^{p_{j}} d \mu_{m-2}(w), \quad z \in \mathbf{U} .
$$

The function $\psi$ is continuous; we can prove the continuity of $\psi$ in a similar fashion as for $\phi_{j}$, observing that $\psi(z), z \in \mathbf{U}$ is uniformly bounded on compact subsets of the unit disc, which follows from the inequality $\psi(z) \leq \prod_{j=1}^{m} \phi_{j}(z)$ since the $\phi_{j}, j=1, \ldots, m$ satisfy this property. Because of continuity, the equality in the first inequality, that is,

$$
\int_{\mathbf{U}} \psi(z) d \mu_{m-2}(z) \leq \int_{\mathbf{U}} \prod_{j=1}^{m} \phi_{j}(z) d \mu_{m-2}(z)
$$

holds (by Proposition 1.1) only if for all $z \in \mathbf{U}$ and some $\zeta^{\prime}(z) \in \mathbf{U}$ and $C_{j}(z) \neq 0$,

$$
f_{j}(z, \cdot)=C_{j}(z) K_{1}^{\frac{2}{p_{j}}}\left(\cdot, \zeta^{\prime}(z)\right), \quad j=1, \ldots, m .
$$

Since $\phi(z) \neq 0, z \in \mathbf{U}$, it is not possible that $f_{j}(z, \cdot) \equiv 0$ for some $j$ and $z$. 
Thus, if equality holds in (1.9), then $f_{j}$ does not vanish, $f_{j}(z, w) \neq 0,(z, w) \in$ $\mathbf{U}^{2}$, and we can obtain some branches $f_{j}^{\frac{p_{j}}{2}}$. Applying Proposition 1.2 for $f_{j}^{\frac{p_{j}}{2}}, j=$ $1, \ldots, m$, we conclude that

$$
f_{j}^{\frac{p_{j}}{2}}=C_{j}^{\prime} K_{2}(\cdot, \zeta), \quad j=1, \ldots, m
$$

must hold for some point $\zeta \in \mathbf{U}^{2}$ and constants $C_{j}^{\prime} \neq 0$. The equality statement of Theorem 1.3 follows.

Remark 2.6. The generalized polydisc is a product $\Omega^{n}=\prod_{k=1}^{n} \Omega_{k} \subset \mathbf{C}^{n}$, where $\Omega_{k}, k=1, \ldots, n$ are simply connected domains in the complex plane with rectifiable boundaries. Let $\partial \Omega^{n}:=\prod_{k=1}^{n} \partial \Omega_{k}$ be its distinguished boundary and let $\phi_{k}: \Omega_{k} \rightarrow$ $\mathbf{U}, k=1, \ldots, n$ be conformal mappings. Then

$$
\Phi(z):=\left(\phi_{1}\left(z_{1}\right), \ldots, \phi_{n}\left(z_{n}\right)\right), \quad z=\left(z_{1}, \ldots, z_{n}\right)
$$

is a bi-holomorphic mapping of $\Omega^{n}$ onto $\mathbf{U}^{n}$.

There are two standard generalizations of Hardy spaces on a hyperbolic simple connected plain domain $\Omega$. One is immediate, by using harmonic majorants, denoted by $H^{p}(\Omega)$. The second is due to Smirnov, usually denoted by $E^{p}(\Omega)$. The definitions can be found in the tenth chapter of the book of Duren [8]. These generalizations coincide if and only if the conformal mapping of $\Omega$ onto the unit disc is a bi-Lipschitz mapping (by [8, Theorem 10.2]); for example, this occurs if the boundary is $C^{1}$ with Dini-continuous normal (Warschawski's theorem; see [35]). The previous can be adapted for generalized polydiscs (see the paper of Kalaj [15]). In particular, $H^{p}\left(\Omega^{n}\right)=E^{p}\left(\Omega^{n}\right)$ and $\|\cdot\|_{H^{p}}=\|\cdot\|_{E^{p}}$ if the distinguished boundary $\partial \Omega^{n}$ is sufficiently smooth, which means that $\partial \Omega_{k}, k=1, \ldots, n$ are sufficiently smooth. Thus, in the case of a sufficiently smooth boundary, we may write

$$
\|f\|_{H^{p}\left(\Omega^{n}\right)}=\left(\frac{1}{(2 \pi)^{n}} \int_{\partial \Omega^{n}}|f(z)|^{p}\left|d z_{1}\right| \ldots\left|d z_{n}\right|\right)^{\frac{1}{p}},
$$

where the integration is carried over the nontangential (distinguished) boundary values of $f \in H^{p}\left(\Omega^{n}\right)$.

By Bremerman's theorem (see [9, Theorem 4.8, pp. 91-93], $E^{2}\left(\Omega^{n}\right)$ is a Hilbert space with the reproducing kernel given by

$$
K_{\Omega^{n}}(z, \zeta):=K_{n}(\Phi(z), \Phi(\zeta))\left(\prod_{k=1}^{n} \phi_{k}^{\prime}\left(z_{k}\right) \overline{\phi_{k}^{\prime}\left(\zeta_{k}\right)}\right)^{\frac{1}{2}}, \quad z, \zeta \in \Omega^{n},
$$

where $K_{n}$ is the reproducing kernel for $H^{2}\left(\mathbf{U}^{n}\right) ; K_{\Omega^{n}}$ does not depend on the particular $\Phi$.

For the next theorem we need the following assertion. The sum $\varphi_{1}+\varphi_{2}$ is a logarithmically subharmonic function in $\Omega$ provided $\varphi_{1}$ and $\varphi_{2}$ are logarithmically subharmonic in $\Omega$ (see e.g. [13, Corollary 1.6.8], or just apply Proposition 2.2 for a discrete measure $\mu$ ). By applying this assertion to the logarithmically subharmonic functions $\varphi_{k}(z)=\left|f_{k}(z)\right|^{2}, z \in \Omega, k=1, \ldots, l$, where $f=\left(f_{1}, \ldots, f_{l}\right)$ is a $\mathbb{C}^{l}$-valued holomorphic function, and the principle of mathematical induction, we obtain that the function $\varphi$ defined by

$$
\varphi(z):=\|f(z)\|=\left(\sum_{k=1}^{l}\left|f_{k}(z)\right|^{2}\right)^{\frac{1}{2}}, \quad z \in \Omega,
$$


is logarithmically subharmonic in $\Omega$ (obviously, the positive exponent of a logarithmically subharmonic function is also logarithmically subharmonic).

Theorem 1.3 in combination with the same approach as in [5] and [15] leads to the following sharp inequality for vector-valued holomorphic functions which generalizes Theorem 3.5 in [5, p. 256]; by vector-valued we mean $\mathbb{C}^{l}$-valued for some integer $l$. We allow vector-valued holomorphic functions to belong to the spaces $H^{p}\left(\Omega^{n}\right)$ if they satisfy the growth condition (1.1) with $\|\cdot\|$ instead of $|\cdot|$.

Let $V_{n}$ be the volume measure in the space $\mathbf{C}^{n}$ and

$$
\lambda_{\Omega^{n}}(z)=K_{n}(\Phi(z), \Phi(z)) \prod_{k=1}^{n}\left|\phi_{k}^{\prime}\left(z_{k}\right)\right|, \quad z \in \Omega^{n}
$$

be the Poincaré metric on the generalized polydisc $\Omega^{n}$ (the right side does not depend on the mapping $\Phi)$.

Theorem 2.7. Let $f_{j} \in H^{p_{j}}\left(\Omega^{n}\right), j=1, \ldots, m$, be holomorphic vector-valued functions on a generalized polydisc $\Omega^{n}$ with sufficiently smooth boundary. The following isoperimetric inequality holds:

$$
\frac{(m-1)^{n}}{\pi^{n}} \int_{\Omega^{n}} \prod_{j=1}^{m}\left\|f_{j}(z)\right\|^{p_{j}} \lambda_{\Omega^{n}}^{2-m}(z) d V_{n}(z) \leq \prod_{j=1}^{m}\left\|f_{j}\right\|_{H^{p_{j}}\left(\Omega^{n}\right)}^{p_{j}} .
$$

For complex-valued functions, the equality in the above inequality occurs if and only if either some of the $f_{j}, j=1, \ldots, m$, are identically equal to zero or if for some point $\zeta \in \Omega^{n}$ and constants $C_{j} \neq 0$ or $C_{j}^{\prime} \neq 0$, the functions have the following form:

$$
f_{j}=C_{j} K_{\Omega^{n}}^{\frac{2}{p_{j}}}(\cdot, \zeta)=C_{j}^{\prime}\left(\prod_{k=1}^{n} \psi_{k}^{\prime}\right)^{\frac{1}{p_{j}}}, \quad j=1, \ldots, m,
$$

where $K_{\Omega^{n}}$ is the reproducing kernel for the domain $\Omega^{n}$ and $\psi_{k}: \Omega_{k} \rightarrow \mathbf{U}, k=$ $1, \ldots, n$, are conformal mappings.

In particular, for $n=1$ and $m=2$ and in the case of complex-valued functions, the above inequality reduces to the result of Mateljević and Pavlović mentioned in the Introduction.

\section{ACKNOWLEDGMENTS}

The author wishes to thank Professors Miodrag Mateljvić and David Kalaj for very useful comments. Also, he is grateful to Professor Darko Mitrović for comments on the exposition and for language corrections.

\section{REFERENCES}

[1] N. Aronszajn, Theory of reproducing kernels, Trans. Amer. Math. Soc. 68 (1950), no. 3, 337-404. MR0051437(14:479c)

[2] E. F. Beckenbach and T. Radó, Subharmonic functions and surfaces of negative curvature, Trans. Amer. Math. Soc. 35 (1933), no. 3, 662-674. MR1501708

[3] C. Bénéteau and D. Khavinson, The isoperimetric inequality via approximation theory and free boundary problems, Comput. Methods Funct. Theory 6 (2006), no. 2, 253-274. MR2291136 (2007i:30001) 
[4] J. Burbea, Inequalities for holomorphic functions of several complex variables, Trans. Amer. Math. Soc. 276 (1983), no. 1, 247-266. MR684506 (84j:32030)

[5] J. Burbea, Sharp inequalities for holomorphic functions, Illinois J. Math. 31 (1987), no. 2, 248-264. MR882113 (89e:32012)

[6] V. Bläsjö, The isoperimetric problem, Amer. Math. Monthly 112 (2005), no. 6, 526-566. MR2142606 (2006a:51017)

[7] T. Carleman, Zur Theorie der Minimalflächen, Math. Z. 9 (1921), no. 1-2, 154-160. MR.1544458

[8] P. L. Duren, Theory of $H^{p}$ Spaces, Academic Press, New York, 1970. MR0268655 (42:3552)

[9] B. A. Fuks, Special Chapters in the Theory of Analytic Functions of Several Complex Variables (Russian), Gosudarstv. Izdat. Fiz.-Mat. Lit., Moscow, 1963. MR0174786 (30:4979)

[10] T. W. Gamelin and D. Khavinson, The isoperimetric inequality and rational approximation, Amer. Math. Monthly 96 (1989), no 1., 18-30. MR.979592 (90d:30108)

[11] P. R. Halmos, Measure Theory, Van Nostrand, New York, 1950. MR0033869 (11:504d)

[12] G. H. Hardy and J. E. Littlewood, Some properties of fractional integrals II, Math. Z. 34 (1932), 403-439. MR:1545260

[13] L. Hörmander, An Introduction to Complex Analysis in Several Variables, North-Holland, Amsterdam, 1973. MR.0344507 (49:9246)

[14] S. Jacobs, An Isoperimetric Inequality for Functions Analytic in Multiply Connected Domains, Mittag-Leffler Institute Report 5 (1972).

[15] D. Kalaj, Isoperimetric inequality for the polydisk, Ann. Mat. Pura Appl. 190 (2011), no. 2, 355-369. MR2786177

[16] D. Kalaj and R. Meštrović, An isoperimetric type inequality for harmonic functions, J. Math. Anal. Appl. 373 (2011), no. 2, 439-448 MR2720695 (2011j:30069)

[17] M. Keldysh and M. Lavrentiev, Sur la représentation conforme des domaines limités par des courbes rectifiables, Ann. Sci. École Norm. Sup. 54 (1937), no. 3, 1-38.

[18] H. O. Kim, On a theorem of Hardy and Littlewood on the polydisc, Proc. Amer. Math. Soc. 97 (1986), no. 3, 403-409. MR840619 (88f:32027)

[19] C. J. Kolaski, Isometries of Bergman spaces over bounded Runge domains, Canad. J. Math. 33 (1981), no. 5, 1157-1164. MR.638372 (83b:32028)

[20] S. G. Krantz, Function Theory of Several Complex Variables, Wiley, New York, 1982. MR635928 (84c:32001)

[21] M. Mateljević, The isoperimetric inequality and some extremal problems in $H^{1}$, Lect. Notes Math. 798, Springer, Berlin, 1980, 364-369. MR577467 (81k:30044)

[22] M. Mateljević, The isoperimetric inequality in the Hardy class $H^{\perp}$, Mat. Vesnik 31 (1979), 169-178. MR613907 (82i:30052)

[23] M. Mateljević and M. Pavlović, New proofs of the isoperimetric inequality and some generalizations, J. Math. Anal. Appl. 98 (1984), no. 1, 25-30. MR.728515 (85c:30001)

[24] M. Mateljević and M. Pavlović, Some inequalities of isoperimetric type concerning analytic and subharmonic functions, Publ. Inst. Math. (Belgrade) (N.S.) 50 (64) (1991), 123-130. MR:1252165 (94g:30032)

[25] M. Mateljević and M. Pavlović, Some inequalities of isoperimetric type for the integral means of analytic functions, Mat. Vesnik 37 (1985), 78-80. MR791569 (86e:30036)

[26] R. Osserman, The isoperimetric inequality, Bull. Amer. Math. Soc. 84 (1978), no. 6, 1182 1238. MR0500557 (58:18161)

[27] M. Pavlović, Introduction to Function Spaces on the Disk, Matematički Institut SANU, Belgrade, 2004. MR2109650 (2006d:30001)

[28] M. Pavlović and M. Dostanić, On the inclusion $H^{2}\left(\mathbb{U}^{n}\right) \subset H^{2 n}\left(\mathbb{B}_{n}\right)$ and the isoperimetric inequality, J. Math. Anal. Appl. 226 (1998), no. 1, 143-149. MR1646501 (99h:46038)

[29] I. I. Privalov, Boundary Properties of Analytic Functions (Russian), Gostekhizdat, Moscow, 1950. MR0047765(13:926h)

[30] L. I. Ronkin, Introduction to the Theory of Entire Functions of Several Variables, Translations of Mathematical Monographs, Amer. Math. Soc., Providence, RI, 1974. MR0346175 (49:10901)

[31] W. Rudin, Function Theory in Polydiscs, Benjamin, New York, 1969. MR0255841 (41:501)

[32] S. Saitoh, The Bergman norm and the Szegö norm, Trans. Amer. Math. Soc. 249 (1979), no. 2, 261-279. MR525673 (80h:30011)

[33] K. Strebel, Quadratic Differentials, Springer-Verlag, Berlin, 1984. MR743423 (86a:30072) 
[34] D. Vukotić, The isoperimetric inequality and a theorem of Hardy and Littlewood, Amer. Math. Monthly 110 (2003), no. 6, 532-536. MR1984405

[35] S. E. Warschawski, On differentiability at the boundary in conformal mapping, Proc. Amer. Math. Soc. 12 (1961), no. 4, 614-620 MR0131524 (24:A1374)

[36] A. Zygmund, Trigonometric Series, Vol. II, 2nd ed., Cambridge University Press, 1959. MR0107776 (21:6498)

Faculty of Natural Sciences and Mathematics, University of Montenegro, Cetinjski Put B.B., 81000, Podgorica, Montenegro

E-mail address: marijanmmarkovic@gmail.com 